\title{
Titel/Title: Reputation statt Wahrheit: Verdrängt der Nebencode den Code?
}

Autor*innen/Author(s): Uwe Schimank

Veröffentlichungsversion/Published version: Verlagsversion (VoR)

Zeitschriftenartikel/Journal article

\section{Empfohlene Zitierung/Recommended citation:}

Schimank, Uwe (2010): Reputation statt Wahrheit: Verdrängt der Nebencode den Code? In: Soziale Systeme, 16 (2), 233-242.

Verfügbar unter/Available at:

(wenn vorhanden, bitte den DOI angeben/please provide the DOI if available)

Die Verlagsveröffentlichung ist verfügbar unter www.degruyter.com https://doi.org/10.1515/sosys-2010-0204

Zusätzliche Informationen/Additional information:

Der Autor kann kontaktiert werden unter: uwe.schimank@uni-bremen.de 


\section{Reputation statt Wahrheit: Verdrängt der Nebencode den Code? ${ }^{1}$}

Fritz Scharpf $(1988,16)$ stößt sich in der völlig unbeachtet gebliebenen Fußnote eines Discussion Papers, in dem er sich kritisch mit Niklas Luhmanns Politikverständnis auseinandersetzt, an »der seltsam inkonsistenten Zuordnung funktionsspezifischer Codes«:

Wenn Wissenschaft durch den Code "Wahr-Unwahr» konstituiert wird, und das Rechtssystem durch "Recht-Unrecht", dann müßste man auf derselben begrifflichen Ebene das politische System durch den Code »Gemeinwohldienlich-Gemeinwohlschädlich « charakterisieren. Wenn umgekehrt die Politik durch die jeweilige Nützlichkeit für »Regierung « oder »Opposition « bestimmt wird, müßte man das Wissenschaftssystem durch "Reputationserwerb " und "Reputationsverlust " definieren, und das Rechtssystem vielleicht durch Sieg oder Niederlage in Rechtsstreitigkeiten. Man kann, so denke ich, den Code entweder durch die funktionsspezifischen "public virtues« oder durch die zugehörigen "private vices« bestimmen, aber nicht hier so und dort anders.

Scharpf sieht hier also eine verzerrte, auch ungerechte Wahrnehmung. Er wirft Luhmann vor, mit zweierlei Maß zu messen. Der Wissenschaft und dem Recht nehme er das hehre Streben nach Wahrheit und Recht ab, während er der Politik - genauer: den Politikern, die bei Luhmann freilich nur als ausgeflaggte Kommunikationsadressen auftauchen können - unterstelle, sich um nichts als Machterhalt und -steigerung zu kümmern.

Ich lasse diese theoriebautechnische Kontroverse einschließlich ihrer beiderseitigen normativen Voreinstellungen hier auf sich beruhen ${ }^{2}$ und frage vielmehr: Könnte dieser Vorbehalt Scharpfs gegen Luhmanns Sicht der Dinge dergestalt von der Wirklichkeit eingeholt worden sein, dass mittlerweile auch die Wissenschaft eindeutig immer stärker von den "private vices« bestimmt oder gar dominiert wird?

1 Für hilfreiche Hinweise danke ich Jochen Gläser, Frank Meier, Lothar Peter und Andreas Stucke.

2 Zum biographischen Entdeckungszusammenhang könnte man ja mit Blick auf Luhmann schon fragen: Wollte er das Geschäft, das er selbst betreibt, nachdem er vorher politiknah in der Ministerialbürokratie gearbeitet hatte, in solch freundliches Licht stellen, weil das dem eigenen Selbstbild zuträglich war? Schließlich kannte er durchaus Taktiken der »Theoriepolitik« (Luhmann 1969, 263-265), die aber in seinem Porträt der »Wissenschaft der Gesellschafte (Luhmann 1990) nicht prominent auftauchen. 


\section{Von der Komplexitätsreduktion für Leser zur Komplexitätsreduktion für Geldgeber}

Luhmann (1968; 1990, 244-251) kennt Reputation durchaus als Nebencode der Wissenschaft. Er gibt ihm freilich eine ziemlich harmonische funktionalistische Interpretation. Weil Wissenschaftler - wieder akteurtheoretisch gelesen - mit der Komplexität dessen, was sie alles lesen könnten, zu kämpfen haben, bietet ihnen die Reputation von Autoren ein Auswahlkriterium. Im Zweifelsfall entscheidet man sich für den bewährten bekannten und gegen den unbekannten Namen. So geht man auf Nummer sicher. Das Risiko, die knappe Zeit für Lektüre auf Schlechtes und Abseitiges verwendet zu haben, wird minimiert. Mit etwas Milieukenntnis lernt man auch, sich nicht länger von »erloschenen Vulkanen« (Luhmann 1984, 13) fesseln zu lassen. Auf die Chance, als Erster etwas Bahnbrechendes eines unbekannten Forschers zu rezipieren und weiter verarbeiten zu können, verzichtet man. Man schätzt angesichts der geringen Wahrscheinlichkeit, im Unbekannten fündig zu werden, den Nutzen, dort zu suchen, für die eigene Wiederverwendung des Gelesenen als geringer ein, als sich von bekannten Namen leiten zu lassen, die zwar selten etwas völlig Neues bieten, aber jedenfalls bewährt Brauchbares.

Der tiefgreifende Governance-Wandel in Richtung "new public management" (NPM), dem die Hochschulsysteme der meisten westlichen Länder eins nach dem anderen unterzogen wurden (de Boer et al. 2007; Schimank/Lange 2009), sorgte nun dafür, dass die Komplexitätsreduktion für Lektüreentscheidungen äußerst folgenreich weitergenutzt wurde. Sie wurde nämlich viel systematischer und rigoroser als zuvor auch zur Grundlage für Finanzierungsentscheidungen gemacht. Die dahinter stehende Logik klingt erst einmal bestechend: Wenn hohe Reputation den Fachkollegen als Indikator für gesicherte Qualität dient, warum sollten sich nicht auch gleichermaßen an Qualität interessierte Geldgeber der Forschung an denselben Reputationskriterien orientieren? Da ja die für die Forschungsfinanzierung verfügbaren staatlichen Finanzmittel seit Mitte der 1970er Jahre zunehmend knapp wurden, sahen sich die Geldgeber gezwungen, verstärkt auf eine leistungsgerechte Mittelallokation zu achten, um knappes Geld nicht bei »Versagern « und »Nichtstuern « zu versenken, sondern möglichst nutzbringend $z u$ investieren.

Zeitgleich waren die Möglichkeiten einer "Vermessung der Forschung (Weingart/Winterhager 1984) entscheidend verbessert worden. Zunächst einmal waren zumindest für die Naturwissenschaften Literaturdatenbanken wie der Science Citation Index aufgebaut worden, die auch die Zitationen von Publikationen recherchierbar machten. Dass dies dann schnell mittels Computern und entsprechender Software unendlich beschleunigt und auch auf die anderen Wissenschaftsgebiete ausgeweitet wurde, sorgte dafür, dass die immer schon als Leistungsmerkmal herangezogene, aber in ihrer Aussagekraft bekanntermaßen begrenzte Länge des Publikationsverzeichnisses gleichsam mit der 
Zitierhäufigkeit jeder Publikation gewichtet werden konnte. Hinzu kam, dass auch das Volumen der von einem Forscher eingeworbenen Drittmittel zum Leistungsmerkmal erhoben wurde - was eigentlich nur aus der Sicht eines Ministeriums, dem die Gelder für die Grundausstattung ausgehen und das sich deshalb über die »Selbsthilfe« seiner Kostgänger freut, und der ebenso kalkulierenden Kanzler der Universitäten Sinn macht. Bis dahin hatten sich deutsche Professoren Drittmittel oft klammheimlich an der Haushaltsabteilung ihrer Hochschule vorbei beschafft, um mehr Flexibilität der Mittelverwendung zu haben. Dies wurde in dem Augenblick sowohl rechtlich unterbunden als auch inopportun, als das Drittmittelvolumen individueller wie organisatorischer Erfolgsindikator wurde und daher in die offizielle Statistik eingehen musste.

Beide Entwicklungen gingen in dieselbe Richtung einer Quantifizierung der Reputation. ${ }^{3}$ Nicht mehr die vielfältigen Eindrücke, die man über jemanden - als Leser von dessen Schriften, als Zuhörer bei dessen Vorträgen, als bei unterschiedlichen Gelegenheiten mit ihm Diskutierender und ihn Beobachtender, nicht zuletzt auch von Hörensagen in Gesprächen über ihn - gewann, ergaben, was man von ihm hielt, sondern Publikationsmengen, impact-Faktoren der Publikationsorte, Zitationshäufigkeiten und Drittmittelsummen. Höchst aufschlussreich sind diesbezüglich Beobachtungen, die man in Berufungskommissionen machen kann. Immer häufiger sind inzwischen auch in den Sozialwissenschaften solche Zahlenwerte, ob nun der Hirsch-Index oder das Gesamtvolumen eingeworbener Drittmittel, geradezu Totschlagargumente, denen man mit Hinweisen darauf, wie raffiniert man das theoretische Modell oder methodische Vorgehen eines - leider nur in einem der notorisch verschrieenen Sammelbände erschienenen - Aufsatzes oder wie gut geschrieben man ein Buch findet, nicht mehr Paroli bieten kann. Davon, dass der Aufsatz so in einem peer-reviewed journal nie erschienen wäre, weil ihn zwei bis drei Überarbeitungsrunden entlang von Gutachterhinweisen zu einem braven Mainstream-Produkt deformiert hätten, und dass nur das Buchformat die anschauliche Dokumentation und Interpretation qualitativer Empirie erlaubt, ganz zu schweigen! Aus Berufungskommissionen von Medizinern, generell den Lebenswissenschaften, aber auch der Volkswirtschaftslehre hört man immer wieder die ernstgemeinte ungeduldige Frage: „Warum diskutieren wir hier eigentlich lange über Stärken und Schwächen von Kandidaten und laden sie gar noch zu einem Probevortrag ein? Wir können doch die einschlägigen Maßzahlen vergleichen und auf der Basis die Berufungsliste erstellen! 4

Das immer schon gegebene Reputationsstreben in der scientific community geht also in immer schärfere Ressourcenkonkurrenz über: Wer kann wo veröffentlichen, wirbt wie viele Drittmittel ein und bekommt welche Stellen mit welcher Ausstattung und welchem Einkommen angeboten?

3 Generell zu quantifizierenden Vergleichen siehe Heintz 2010.

4 Die teilweise horrenden Fehler, die diese zahlengläubige »amateur bibliometrics «egeht, sind ein Kapitel für sich (Gläser/Laudel 2007, 116-118). 


\section{Von der Komplexitätsreduktion für andere zur eigenen Handlungsorientierung}

Je spezifischer die Reputationskriterien durch Quantifizierung geworden sind, desto mehr ist es dem Wissenschaftler nicht nur möglich geworden, diese Kriterien als Orientierungsgrößen eigenen Forschungshandelns zu nehmen - angesichts der Konkurrenzintensivierung sieht er dies auch als geboten an. Der »Homo academicus « (Bourdieu 1984), der sich stets auch strategisch um die eigene Reputation kümmert, damit er sie zur Ressourcenmobilisierung für weitere Forschung einsetzen kann, ${ }^{5}$ war immer schon ein Teil der Wissenschaftler-Identität und auch früher bei Einigen der Identitätskern. Doch nun droht er sich, zum »Homo academicus oeconomicus « (Peter 2010) dressiert, flächendeckend breit zu machen, was dem traditionellen Gelehrten, der zuallererst von »curiositas « getrieben wird und die eigene Reputationssteigerung allenfalls als nicht ganz außer Acht zu lassende Konzession an die Spielregeln der scientific community ansieht, endgültig den Lebensraum nehmen könnte. Inwiefern kann diese Zielverschiebung von Wahrheitssuche zu Reputationsstreben dem wissenschaftlichen Erkenntnisfortschritt abträglich sein? Die Dogmatiker des NPM versprechen ja genau das Gegenteil: Die Qualität der Forschung steige, wenn die Wissenschaftler auf der Basis von Evaluationen mittels standardisierter Indikatoren und sich damit verknüpfender Ressourcenzuteilungen unter Konkurrenzdruck gesetzt werden. Doch eine ganze Reihe von Überlegungen und teilweise bereits vorliegenden Beobachtungen sprechen eine deutlich andere Sprache:

- Am augenfälligsten ist die offenkundige Zunahme von - aufgedeckten Betrugsfällen (Mayntz 2000), also insbesondere der Fälschung empirischer Daten, um theoretische Behauptungen beweisen zu können. Fälschung beginnt bereits dort, wo uneindeutige Daten so »frisiert« werden, dass sie den Anschein glatter Bestätigungen erwecken. Ziel dabei ist, an guten Orten publizieren zu können und sich günstige Förderchancen für Fortsetzungsprojekte zu verschaffen. Da das peer review bei der Manuskriptbegutachtung bekannte Schwächen hat, die meisten Publikationen - auch in den top journals - gar nicht oder nur oberflächlich gelesen und die zugrundeliegenden empirischen Studien nur selten repliziert werden, weil man damit keine Reputation erwerben kann, ist das Risiko, des Betrugs überführt zu werden, gering. Das heißt zwar einerseits, dass Fälschungen oftmals keinen unmittelbaren Schaden anrichten, weil sie schlicht von der weiteren Forschung gar nicht zur Kenntnis genommen werden. Andererseits ist der mittelbare Schaden beträchtlich: Knappe Publikationsorte und Fördermittel werden de-

5 Siehe Braun (1994, 66-83), der insbesondere auf Latour/Woolgar (1979) zurückgreift. 
nen vorenthalten, die ehrlich, aber dafür weniger spektakulär forschen. Dies war zwar schon immer so - aber je größer sowohl der Konkurrenzdruck als auch die Knappheiten werden, desto größer wachsen sich auch diese dysfunktionalen Effekte aus. Dies erst recht, je mehr sich in der Szene herumspricht, dass Betrug als Praxis einer Anpassung durch Abweichung weit verbreitet sei, wodurch dies zur sich selbst erfüllenden Prophezeiung wird.

- Weniger dramatisch, aber dafür umso verbreiteter sind suboptimale Forschungspraktiken, die insbesondere auf Zeitdruck sowie auf fehlende Ressourcen zurückgehen, mit der Folge eines Verlusts an Zuverlässigkeit der Forschungsergebnisse (Gläser et al. 2008, 161/162, 166). Hierzu gehören etwa die schludrige Durchführung von Experimenten oder Tests sowie zweit- oder drittbeste Versuchsanordnungen, weil z. B. nur veraltete Messgeräte vorhanden sind. Auch der Einsatz unzulänglich angeleiteter Hilfskräfte oder Doktoranden - weil die erfahrenen Wissenschaftler völlig mit dem Schreiben von Anträgen und der Netzwerkpflege eingedeckt sind - ist ein Phänomen in Kauf genommener Suboptimalität, das seit längerem schon um sich greift; und wiederum stehen Knappheiten und Konkurrenzdruck dahinter.

- Viel gesprochen wird mittlerweile auch über sachlich suboptimale Publikationspraktiken, die im Zuge von quantifizierten Reputationszuteilungen üblich werden (Enders et al. 2011). Die bekannte Salami-Taktik der Fragmentierung von Forschungsergebnissen in »least publishable units« gehört ebenso dazu wie die doppelte und dreifache Veröffentlichung derselben Erkenntnis unter anderen Titeln und an anderen Orten. Auch die Neigung, den Publikationsort für Forschungsergebnisse nicht mehr primär unter dem Gesichtspunkt zu wählen, wo die zentral daran interessierten peers am ehesten darauf stoßen, sondern sich unter allen erreichbaren Publikationsorten für den mit dem höchsten Impact-Faktor zu entscheiden, also etwa für »Nature « statt für das einschlägige hochgradig spezialisierte Journal, steigert zwar die individuelle Reputation, ist aber eher dysfunktional für das kollektive Streben nach Erkenntnisfortschritt. Insbesondere Geisteswissenschaftler haben darüber hinaus zur Kenntnis zu nehmen, dass die Monographie in standardisierten Evaluationen viel zu gering geschätzt wird, obwohl typische Themenstellungen dieser Disziplinen sie als nach wie vor wichtigste Publikationsart ausweisen.

- Das durch Knappheiten und Konkurrenzdruck auferlegte Reputationsstreben führt weiterhin zu einer Verengung des Forschungs-Portfolios der Wissenschaftler und einer Kurzatmigkeit ihrer Forschungsaktivitäten (Gläser et al. 2008, 160-162, 165). Immer geringere Ressourcen 
müssen gebündelt werden und tragen keine simultan vorangetriebenen Forschungslinien mehr, womit die wechselseitige Befruchtung, auch im Sinne von »serendipity « (Merton 1948, 100-104), also Gelegenheitsstrukturen für Zufallsfunde, wegfällt. Längerfristig angelegte Forschungslinien werden immer mehr erschwert und müssen häufiger auf halbem Wege abgebrochen werden, weil Förderprioritäten nicht selten wie Moden wechseln und die Projektförderung generell auf kürzere Formate angelegt wird, wobei stets unsicher ist, ob ein Anschlussprojekt bewilligt wird oder nicht.

- Die standardisierten Evaluationskriterien laufen schnell auf eine »one size fits all«-Politik hinaus, die dann im schlimmsten Fall z.B. einen Mathematiker dazu zwingt, zum Wohle seines Fachbereichs und seiner Universität Drittmittel einzuwerben, obwohl er von den Forschungsfragen seines Teilgebiets her sinnvoller Weise Einzelforschung betreiben müsste. Stattdessen hält ihn für die nächsten drei Jahre die Betreuung zweier Projektmitarbeiter vom Nachdenken über seine eigenen Fragen $a b$, ohne dass deren Fragen ihn weiterbrächten. Eine inzwischen insbesondere in Deutschland verbreitete Spielart dieser Fehlsteuerung findet sich in der geradezu zur fixen Idee gewordenen Vorstellung von Forschungspolitikern und Hochschulleitungen, dass gute Forschung heutzutage nur noch in Gestalt »kritischer Massen « kooperierender Forscher, also in Clustern, Schwerpunkten, Verbünden etc. produziert werden könne (Schiene/Schimank 2007). Die »Exzellenzinitiative« ist das sichtbarste Aushängeschild dieser forschungspolitischen Leitidee. Neben sachlich gebotenen und ertragreichen Kooperationen werden auch Zweckbündnisse zusammengetrieben, die von Anfang an nichts als allseitige Quälerei sind, wenn Forschungspolitik und Hochschulleitungen, ihrerseits unter Vorzeigedruck stehend, entsprechende Leistungsindikatoren formulieren.

- Ein ohnehin immer schon angelegter Drang von Wissenschaftlern zum sicheren Mainstream wird durch Knappheiten und Konkurrenzdruck dysfunktional verstärkt. Wer dringender denn je Drittmittel braucht, wird sich opportunistisch gemutmaßten Vorlieben von Gutachtern und Förderorganisationen anzupassen versuchen; und wer auf Gedeih und Verderb in die wichtigen Zeitschriften hinein will, muss sich der von Jochen Gläser (2006, 125-130) so charakterisierten Zurichtung seines Manuskripts durch peer review beugen: »der Autor gibt so weit nach, wie er muss, um eine Publikation zu erreichen.« (129) Unorthodoxe Forschungsperspektiven bleiben so auf der Strecke, was auch dann, wenn viele davon abstrus sind, höchst dysfunktional sein kann, weil irgendwann nur noch ganz andere Ideen zum Wagnis animieren können, die ausgetretenen Pfade des Mainstream hinter sich zu lassen. 
- Mit je größeren finanziellen Konsequenzen man die zwangsläufig selektiven standardisierten Leistungsindikatoren verbindet, desto mehr ruft man dadurch auf Seiten der Wissenschaftler eine systematische Vernachlässigung davon nicht erfasster, aber gleichwohl unentbehrlicher Teil-Aktivitäten der wissenschaftlichen Leistungsproduktion hervor. Wenn - im Extremfall - allein »Drittmittelkönige« und »Vielpublizierer« belohnt werden und diese Belohnungen entsprechend mehr Gewicht bekommen, dann darf man sich nicht darüber wundern, dass die Unterstützung des Nachwuchses, das Rezensionswesen, die Herausgabe von Fachzeitschriften und Buchreihen, das Gutachtenschreiben, die Organisation von Tagungen und Kongressen sowie die öffentliche Vermittlung der fachlichen Erkenntnisse u. ä. links liegen bleiben (Jansen 2009, 47-49).

NPM entpuppt sich also als ein ausgesprochen heikles Unterfangen. Zwar kann man mittels einer Entfesselung von Ressourcenkonkurrenz anhand quantifizierter Leistungskriterien die quantitative und qualitative Produktivität wissenschaftlicher Wahrheitssuche wohl durchaus erst einmal ein Stück weit verbessern, indem Leistungsschwache zu erhöhter Anstrengung getrieben oder ihnen, sollte das nicht wirken, die Ressourcen entzogen werden und bei Leistungsstärkeren bessere Verwendung finden. Insoweit liegen die Wissenschaftspolitiker und Hochschulleitungen richtig. Aber sehr schnell hat man diese Stellschraube zu stark angezogen, was dann all die aufgelisteten Dysfunktionalitäten mit sich bringt.

\section{Quantifizierung als Verlust der »occupational control «}

Die Problematik reicht allerdings noch weiter. In dem Moment, in dem Wissenschaftler es zulassen oder hinnehmen müssen, dass ihre Leistungen anhand von Kennziffern bewertet werden, die keiner weiteren Interpretation, wie sie nur Angehörige der betreffenden Disziplin selbst geben können, mehr zu bedürfen scheinen, geben sie einen essentiellen Garanten ihrer »occupational control « im Sinne einer "collective capability of members of an occupation to preserve unique authority in the definition, conduct, and evaluation of their work « (Child/Fulk 1982, 155) auf. Wenn Wissenschaftler selbst die Qualität ihrer Kollegen nur noch anhand von Zitationsraten, impact-Faktoren und Drittmittelsummen beurteilen und auf das Lesen und Abwägen verzichten zu können meinen, ${ }^{6}$ dürfen sie sich nicht wundern, wenn Forschungspolitiker, Rektoren, Kanzler oder die Administratoren der Förderorganisationen im Brustton der Überzeugung sagen: „Das können wir auch!« 
Es ist eine gewisse Ironie der Geschichte, dass sich mit den Naturwissenschaften ausgerechnet diejenigen Disziplinen, die sich eine besonders gehärtete Wissenschaftlichkeit und damit auch Autonomie gegen außerwissenschaftliche Einflüsse zusprechen, am weitesten auf diesen Weg begeben haben, während die Sozialwissenschaften noch zögern und die Geisteswissenschaften sich vehement verweigern. So konnte man es beim Forschungsrating des Wissenschaftsrats beobachten: Die Chemiker hatten wenig Probleme damit, standardisierte Kennziffern für sich sprechen zu lassen; die Soziologen beharrten hingegen darauf, noch Schriftproben der zu Beurteilenden zu lesen und diese Eindrücke neben und immer wieder auch gegen die Zahlen zu stellen; und die Historiker erklärten rundheraus, die Qualität ihrer Forschungen ließe sich nicht quantitativ-standardisiert vermessen, und nahmen am Rating nicht teil. Die Frage ist nur, welche Linie sich längerfristig durchsetzen wird. Spätestens dann jedenfalls, wenn vorrangig auf Grundlage solcher quantitativer Leistungsbewertungen Ressourcen vergeben werden, regiert der Nebencode der Wissenschaft. Die äußere Form einer Autopoiesis der Publikationen (Stichweh 1987) als wissenschaftsspezifische Manifestation teilsystemischer Autonomie bleibt zwar gewahrt. Aber wenn Wissenschaftler nicht länger vor allem anderen den Streit in der Sache suchen, sondern sich bemühen, denjenigen Kollegen, die über begehrte Publikationsorte und Drittmittel entscheiden, nach dem Munde zu schreiben sowie darüber hinaus möglichst vielen anderen, die man zu Zitationen animieren will, zu gefallen, wird Wahrheitsstreben durchgängig opportunistischen Erwägungen der Reputationssteigerung unterworfen. Nicht, dass das nicht immer schon in gewissem Maße mitgelaufen wäre und sich bisweilen auch früher schon verselbständigt hätte! Aber wenn das der Regelfall wird, leidet die Wahrheit. Überspitzt gesagt: Wenn niemand mehr irgendjemanden, außer einige auserkorene Sündenböcke, in der Sache herausfordert, um nicht anzuecken, versinken alle, über braves wechselseitiges Zitieren autopoietisch aneinandergekettet, gemeinsam im Sumpf des ideenfeindlichen Mittelmaßes - was freilich so schnell keiner merkt, weil ja andere, bessere Wahrheiten gar nicht mehr hervorgebracht werden und die äußerliche Bilanz der Publikationsmengen, Zitationsraten und Drittmittelsummen weiterhin stimmt, sich wahrscheinlich sogar noch verbessert.?

7 Wenn die Wissenschaftsforschung, insbesondere in ihrer systemtheoretischen Fassung bei Luhmann oder Stichweh, Autonomiegefährdungen der Wissenschaft durch die Politik reflektiert, stellt sie sich vor allem vor, dass der Forschung politische Gesichtspunkte gesellschaftlicher »relevance« aufgedrängt würden. Aber heutzutage ist das weitaus gewichtigere Problem, dass die Wissenschaftspolitik ja nur das Beste für die Wissenschaft - nämlich die Qualitätsverbesserung der Forschung gemäß innerwissenschaftlichen Gütemaßen - will und im festen Glauben ist, sie wisse das im Zweifelsfalle am besten und könne es vor allem besser durchsetzen als die scientific community selbst. Das ist - bei Gleichheit in der oberflächlichen Betrachtung und in der Selbstwahrnehmung - tatsächlich das genaue Gegenteil der "preußischen Bildungsverwaltung, die sich ... durch einen ungewöhnlichen Grad der Internalisierung akademischer Selbstverständnisse auszeichnete " (Stichweh 2009, 48). 


\section{Literatur}

Bourdieu, Pierre (1984): Homo Academicus. Frankfurt a. M.: Suhrkamp.

Braun, Dietmar (1994): Die politische Steuerung der Wissenschaft. Ein Beitrag zum »kooperativen Staat«. Frankfurt a. M.: Campus.

Child, John / Fulk, Janet (1982): Maintenance of Occupational Control. The Case of Professions. Work and Occupations 9, 155-192.

de Boer, Harry/Enders, Jürgen/Schimank, Uwe (2007): On the Way towards New Public Management? The Governance of University Systems in England, the Netherlands, Austria, and Germany. S. 137-152 in: Dorothea Jansen (Hrsg.), New Forms of Governance in Research Organizations. Disciplinary Approaches, Interfaces and Integration. Dordrecht: Springer.

Enders, Jürgen/Kehm, Barbara/Schimank, Uwe (2011): Turning Universities into Actors on Quasi-Markets: How New Public Management Reforms Affect Academic Research. In: Dorothea Jansen/Insa Pruisken (Hrsg.), The changing governance of higher education and research - multilevel perspectives. Dordrecht: Springer (im Erscheinen).

Gläser, Jochen (2006): Wissenschaftliche Produktionsgemeinschaften. Die soziale Ordnung der Forschung. Frankfurt a. M.: Campus.

Gläser, Jochen/Lange, Stefan/Laudel, Grit/Schimank, Uwe (2008): Evaluationsbasierte Forschungsfinanzierung und ihre Folgen. S. 145-170 in: Renate Mayntz et al. (Hrsg.), Wissensproduktion und Wissenstransfer - Wissen im Spannungsfeld von Wissenschaft, Politik und Öffentlichkeit. Bielefeld: transcript.

Gläser, Jochen / Laudel, Grit (2007): The Social Construction of Bibliometric Evaluation. S. 101-123 in: Richard Whitley/Jochen Gläser (Hrsg.), The Changing Governance of the Sciences. The Advent of Research Evaluation Systems. Dordrecht: Springer.

Heintz, Bettina (2010): Numerische Differenz. Überlegungen zu einer Soziologie des (quantitativen) Vergleichs. Zeitschrift für Soziologie 39, 162-181.

Jansen, Dorothea (2009): Neue Governance im deutschen Forschungssystem. Umsetzung und Wirkungen auf der Arbeitsebene der Forschung. S. 37-59 in: Dorothea Jansen (Hrsg.), Neue Governance für die Forschung. Baden-Baden: Nomos.

Latour, Bruno/Woolgar, Steve (1979): Laboratory Life. The Social Construction of Scientific Facts. Beverly Hills: Sage.

Laudel, Grit/Gläser, Jochen (2008): Responses to the Loss of Peerness in Evaluations. Paper presented at the Joint 4S/EASST Conference »Acting with Science, Technology, and Medicine $\ll$, Rotterdam, 20-23 August 2008.

Luhmann, Niklas (1968): Selbststeuerung der Wissenschaft. S. 232-252 in: Niklas Luhmann, Soziologische Aufklärung, Bd. 1: Aufsätze zur Theorie sozialer Systeme. Opladen: Westdeutscher Verlag, 1974.

Luhmann, Niklas (1969): Die Praxis der Theorie. S. 253-267 in: Niklas Luhmann, Soziologische Aufklärung, Bd. 1: Aufsätze zur Theorie sozialer Systeme. Opladen: Westdeutscher Verlag, 1974.

Luhmann, Niklas (1984): Soziale Systeme. Grundriß einer allgemeinen Theorie. Frankfurt a. M.: Suhrkamp.

Luhmann, Niklas (1990): Die Wissenschaft der Gesellschaft. Frankfurt a. M.: Suhrkamp.

Mayntz, Renate (2000): Wissenschaftliches Fehlverhalten: Formen, Faktoren und Unterschiede zwischen Wissenschaftsgebieten. S. 57-72 in: Max-Planck-Forum 2, Ethos der Forschung. München: Max-Planck-Gesellschaft.

Merton, Robert K. (1948): Der Einfluß der empirischen Forschung auf die soziologische Theorie. S. 83-113 in: Robert K. Merton, Soziologische Theorie und soziale Struktur. Berlin: de Gruyter, 1995.

Peter, Lothar (2010): Der Homo academicus. S. 206-218 in: Stephan Moebius/Markus Schroer (Hrsg.), Diven, Hacker, Spekulanten. Sozialfiguren der Gegenwart. Frankfurt a. M.: Suhrkamp. 
Scharpf, Fritz (1988): Verhandlungssysteme, Verteilungskonflikte und Pathologien der politischen Steuerung. Köln: Max-Planck-Institut für Gesellschaftsforschung, Discussion Paper 88/1.

Schiene, Christof/Schimank, Uwe (2007): Research Evaluation as Organizational Development: The Work of the Academic Advisory Council in Lower Saxony (FRG). S. 171-190 in: Richard Whitley/ Jochen Gläser (Hrsg.), The Changing Governance of the Sciences: The Advent of Research Evaluation Systems, Dordrecht: Springer.

Schimank, Uwe/Lange, Stefan (2009): Germany: A Latecomer to New Public Management. S. 51-75 in: Catherine Paradiese et al. (eds.), University Governance - Western European Comparative Perspectives. Dordrecht: Springer.

Stichweh, Rudolf (1987): Die Autopoiesis der Wissenschaft. S. 447-481 in: Dirk Baecker et al. (Hrsg.), Theorie als Passion. Niklas Luhmann zum sechzigsten Geburtstag. Frankfurt a. M.: Suhrkamp.

Stichweh, Rudolf (2009): Autonomie der Universitäten in Europa und Nordamerika. Historische und systematische Überlegungen. S. 38-49, 91-92 in: Jürgen Kaube (Hrsg.), Die Illusion der Exzellenz. Berlin: Wagenbach.

Weingart, Peter/Winterhager, Matthias (1984): Die Vermessung der Forschung. Theorie und Praxis der Wissenschaftsindikatoren. Frankfurt a. M.: Campus.

Prof. Dr. Uwe Schimank

Institut für Soziologie, Universität Bremen

Postfach 3304 40, D-28359 Bremen

uwe.schimank@uni-bremen.de 